\title{
Function spaces induced by two parabolic Bloch spaces
}

\author{
Yôsuke HishiKawa, Masaharu Nishio, Katsunori Shimomura and \\ Masahiro YAMADA \\ (Received April 26, 2020) \\ (Revised February 19, 2021)
}

\begin{abstract}
We consider function spaces which consist of two parabolic Bloch spaces, and present reproducing formulas. As an application, we introduce Bloch type spaces which consist of solutions of a partial differential equation $\left(L^{(\alpha)}\right)^{2} u=0$, and investigate several properties.
\end{abstract}

\section{Introduction}

Let $H$ be the upper half-space of the $(n+1)$-dimensional Euclidean space $\mathbb{R}^{n+1}(n \geq 1)$, that is, $H=\left\{(x, t) \in \mathbb{R}^{n+1} ; x \in \mathbb{R}^{n}, t>0\right\}$. For $0<\alpha \leq 1$, the parabolic operator $L^{(\alpha)}$ is defined by

$$
L^{(\alpha)}=\partial_{t}+\left(-\Delta_{x}\right)^{\alpha},
$$

where $\partial_{t}=\partial / \partial t$ and $\Delta_{x}$ is the Laplacian with respect to $x$. Let $C(H)$ be the set of all real-valued continuous functions on $H$, and $C^{k}(H)$ the set of all $k$ times continuously differentiable functions on $H$. A function $u \in C(H)$ is said to be $L^{(\alpha)}$-harmonic if $L^{(\alpha)} u=0$ in the sense of distributions (for details, see Section 2).

We describe the definition of parabolic Bloch spaces. Put $m(\alpha)=$ $\min \left\{1, \frac{1}{2 \alpha}\right\}$. Let $\sigma>-m(\alpha)$. We denote by $\mathscr{B}_{\alpha}(\sigma)$ the set of all $L^{(\alpha)}$-harmonic functions $u \in C^{1}(H)$ which satisfy

$$
\|u\|_{\mathscr{B}_{\alpha}(\sigma)}:=\sup _{(x, t) \in H} t^{\sigma}\left\{t^{1 / 2 \alpha}\left|\nabla_{x} u(x, t)\right|+t\left|\partial_{t} u(x, t)\right|\right\}<\infty,
$$

where $\nabla_{x}=\left(\partial_{1}, \ldots, \partial_{n}\right)$ and $\partial_{j}=\partial / \partial x_{j}$. We also denote by $\tilde{\mathscr{B}}_{\alpha}(\sigma)$ the set of all functions $u \in \mathscr{B}_{\alpha}(\sigma)$ which satisfy $u(0,1)=0$. We call $\tilde{\mathscr{B}}_{\alpha}(\sigma)\left(\right.$ or $\left.\mathscr{B}_{\alpha}(\sigma)\right)$ the parabolic Bloch space. We remark that $\tilde{\mathscr{B}}_{\alpha}(\sigma)$ is a Banach space with the

The Second and Third authors are supported by Grant-in-aid for Science Research, No. JP19K03523.

The Fourth author is supported by Grant-in-aid for Science Research, No. JP16K05198.

2010 Mathematics Subject Classification. Primary 35K05; Secondary 30H30, 26A33.

Key words and phrases. parabolic operator of fractional order, Bloch space, reproducing kernel. 
norm $\|\cdot\|_{\mathscr{B}_{\alpha}(\sigma)}($ see $[2])$. Furthermore, we also note that $\tilde{\mathscr{B}}_{1 / 2}(0)$ coincides with the harmonic Bloch space of Ramey and $\mathrm{Yi}$ [9].

Our aim of this paper is to study function spaces which consist of polyharmonic functions and solutions of the iterative parabolic equation $\left(L^{(\alpha)}\right)^{m} u=$ 0 (called poly-parabolic functions). We state the background of this study. Pavlović [8] introduced the Almansi type decomposition for Hardy spaces of poly-harmonic functions, and investigated several properties. Tanaka [10] introduced bi-harmonic Bergman spaces on the unit ball in $\mathbb{R}^{n}$, and investigated the reproducing kernel, which was given by the Almansi type decomposition. After that, Nishio and Shimomura [5] introduced an iterated parabolic operator $\left(L^{(\alpha)}\right)^{m}$ and poly-parabolic Bergman spaces $\boldsymbol{b}_{\alpha}^{m, p}$. They also gave the Almansi type decomposition with respect to poly-parabolic Bergman functions, and characterized the reproducing kernel on the Hilbert space $\boldsymbol{b}_{\alpha}^{m, 2}$. On the other hand, there is no similar discussion with respect to bi-parabolic or poly-parabolic Bloch type functions. It is known that the investigation of Bloch type spaces plays an important role for the study of Bergman type spaces. Therefore, we introduce Bloch type spaces which consist of bi-parabolic functions, and investigate several properties. In this paper, we consider a kind of sum spaces which consist of two parabolic Bloch spaces, which is based on the study of bi-parabolic or poly-parabolic function theory. And we investigate the reproducing formula on sum spaces. After that, we derive bi-parabolic Bloch spaces from parabolic Bloch spaces, and discuss dualities.

Here, we describe a remark of this paper. We will be able to consider a natural extension to a theory of poly-parabolic Bloch spaces. We just have to introduce sum spaces which consist of several parabolic Bloch spaces. And we can see that the investigation of poly-parabolic Bloch spaces is similar to that of bi-parabolic Bloch spaces.

To state our result, we give some notations. We denote by $d V(x, t)=$ $d x d t$ the Lebesgue volume measure on $H$. Also, we denote by $W^{(\alpha)}$ the fundamental solution of $L^{(\alpha)}$. It is well known that the fundamental solutions of $L^{(1 / 2)}$ and $L^{(1)}$ are the Poisson kernel and the heat kernel, respectively. For a real number $\kappa$, we denote by $\mathscr{D}_{t}^{\kappa}=\left(-\partial_{t}\right)^{\kappa}$ a differential operator of fractional order $\kappa$ (for the explicit definition, see Section 2).

We introduce the following function spaces which consist of parabolic Bloch functions.

Definition 1. Let $0<\alpha \leq 1, \sigma>-m(\alpha)$, and $\rho \neq 0$ be real numbers such that $\sigma+\rho>-m(\alpha)$. A function space $\mathscr{B}_{\alpha}(\sigma, \rho)$ consists of all functions $u$ on $H$ which satisfy $u(x, t)=u_{0}(x, t)+t^{\rho} u_{1}(x, t)$, where $u_{0} \in \tilde{\mathscr{B}}_{\alpha}(\sigma)$ and $u_{1} \in$ $\tilde{\mathscr{B}}_{\alpha}(\sigma+\rho)$. 
We remark that $\mathscr{B}_{\alpha}(\sigma, \rho)$ is a Banach space under the norm $\|\cdot\|_{\mathscr{B}_{\alpha}(\sigma, \rho)}$ defined by

$$
\|u\|_{\mathscr{B}_{\alpha}(\sigma, \rho)}=\left\|u_{0}\right\|_{\mathscr{B}_{\alpha}(\sigma)}+\left\|u_{1}\right\|_{\mathscr{B}_{\alpha}(\sigma+\rho)}
$$

The quantity $\|u\|_{\mathscr{B}_{\alpha}(\sigma, \rho)}$ is independent of the choices $u_{0}$ and $u_{1}$. In fact, it is easy to show that

$$
\tilde{\mathscr{B}}_{\alpha}(\sigma) \cap\left\{t^{\rho} u ; u \in \tilde{\mathscr{B}}_{\alpha}(\sigma+\rho)\right\}=\{0\},
$$

because $u$ and $t^{\rho} u$ are $L^{(\alpha)}$-harmonic on $H$ if and only if $u \equiv 0$.

Now, we present the main results of this paper. The first result is the reproducing formula on $\mathscr{B}_{\alpha}(\sigma, \rho)$. For a function $u(x, t)=u_{0}(x, t)+t^{\rho} u_{1}(x, t)$ on $H$ and a real number $\kappa$, we define a differential operator $\mathscr{E}_{t} \kappa$ by

$$
\mathscr{E}_{t}{ }^{\kappa} u(x, t)=\mathscr{D}_{t}{ }^{\kappa} u_{0}(x, t)+t^{\rho} \mathscr{D}_{t}{ }^{\kappa} u_{1}(x, t)
$$

THEOREM 1. Let $0<\alpha \leq 1, \sigma>-m(\alpha)$, and $\rho>0$ such that $\sigma+\rho>0$. Also, let $\kappa>\max \{0,-\sigma\}$ and $v>\sigma$. Then, the reproducing formula

$$
u(x, t)=2^{v+\kappa} \int_{H} \mathscr{E}_{t}^{\kappa} u(y, s) \mathscr{R}_{\alpha}^{v, \kappa, \rho}(x, t ; y, s) s^{v+\kappa-1} d V(y, s)
$$

holds for all $u \in \mathscr{B}_{\alpha}(\sigma, \rho)$ and $(x, t) \in H$, where

$$
\begin{aligned}
& \mathscr{R}_{\alpha}^{v, \kappa, \rho}(x, t ; y, s)=\sum_{k, \ell=0}^{1} C_{k, \ell} 2^{(k+\ell) \rho} t^{k \rho} s^{\ell \rho} \omega_{\alpha}^{v+(k+\ell) \rho}(x, t ; y, s), \\
& \omega_{\alpha}^{v}(x, t ; y, s)=\mathscr{D}_{t}^{v} W^{(\alpha)}(x-y, t+s)-\mathscr{D}_{t}^{v} W^{(\alpha)}(-y, 1+s),
\end{aligned}
$$

and

$$
\left(\begin{array}{ll}
C_{0,0} & C_{0,1} \\
C_{1,0} & C_{1,1}
\end{array}\right)\left(\begin{array}{cc}
\Gamma(v+\kappa) & \Gamma(v+\kappa+\rho) \\
\Gamma(v+\kappa+\rho) & \Gamma(v+\kappa+2 \rho)
\end{array}\right)=\left(\begin{array}{ll}
1 & 0 \\
0 & 1
\end{array}\right) .
$$

The second result is a characterization of dual spaces with respect to biparabolic Bergman spaces. We denote by $\boldsymbol{b}_{\alpha}^{2,1}(\lambda)$ the bi-parabolic Bergman space with a weighted Lebesgue volume measure $t^{\lambda} d V$, which was introduced in [6] (for explicit definition, see Section 5). Moreover, we define the integral pairing on $\boldsymbol{b}_{\alpha}^{2,1}(\lambda) \times \mathscr{B}_{\alpha}(\sigma, 1)$ by

$$
\langle u, v\rangle_{\lambda, \sigma}=2^{\lambda+\sigma+2} \int_{H} u(x, t) \mathscr{E}_{t} v(x, t) t^{\lambda+\sigma+1} d V(x, t), \quad u \in \boldsymbol{b}_{\alpha}^{2,1}(\lambda), v \in \mathscr{B}_{\alpha}(\sigma, 1),
$$

where $\mathscr{E}_{t}=\mathscr{E}_{t}^{1}$. We claim that a dual space of $\boldsymbol{b}_{\alpha}^{2,1}(\lambda)$ is isomorphic to $\mathscr{B}_{\alpha}(\sigma, 1)$ under a suitable integral pairing. 
THeOrem 2. Let $0<\alpha \leq 1, \lambda>-1$, and $\sigma>-m(\alpha)$. Then, $\left(\boldsymbol{b}_{\alpha}^{2,1}(\lambda)\right)^{*} \cong$ $\mathscr{B}_{\alpha}(\sigma, 1)$ under the pairing

$$
\Phi_{v}(u)=\langle u, v\rangle_{\lambda, \sigma}, \quad u \in \boldsymbol{b}_{\alpha}^{2,1}(\lambda),
$$

where $\Phi_{v}$ is the linear functional induced by $v \in \mathscr{B}_{\alpha}(\sigma, 1)$. Moreover, there exists a constant $C>0$ independent of $v$ such that

$$
C^{-1}\|v\|_{\mathscr{B}_{\alpha}(\sigma, 1)} \leq\left\|\Phi_{v}\right\| \leq C\|v\|_{\mathscr{B}_{\alpha}(\sigma, 1)} .
$$

Finally, we describe the construction of this paper. In Section 2, we give some notations, and present basic properties which are used in our argument. In Section 3, we present the previous result with respect to parabolic Bloch functions. And we investigate a generalization of the reproducing formula of Lemma 5 below. In Section 4, we introduce a function space $\mathscr{B}_{\alpha}(\sigma, \rho)$, and study properties of $\mathscr{B}_{\alpha}(\sigma, \rho)$-functions. In particular, we prove the reproducing formula on $\mathscr{B}_{\alpha}(\sigma, \rho)$. As an application, we discuss dual and pre-dual spaces of bi-parabolic Bergman spaces in Section 5.

\section{Preliminaries}

In this section, we present basic properties of fractional calculus of $L^{(\alpha)}$ harmonic functions. We describe the definition of $L^{(\alpha)}$-harmonic functions. Since the case $\alpha=1$ is trivial, we only describe the case $0<\alpha<1$. Let $C^{\infty}(H)$ be the set of all infinitely differentiable functions on $H$ and $C_{c}^{\infty}(H)$ the set of all functions in $C^{\infty}(H)$ with compact support. For $0<\alpha<1,\left(-\Delta_{x}\right)^{\alpha}$ is the convolution operator defined by

$$
\left(-\Delta_{x}\right)^{\alpha} \psi(x, t)=-c_{n, \alpha} \lim _{\delta \rightarrow 0^{+}} \int_{|y-x|>\delta} \frac{\psi(y, t)-\psi(x, t)}{|y-x|^{n+2 \alpha}} d y
$$

for all $\psi \in C_{c}^{\infty}(H)$ and $(x, t) \in H$, where

$$
c_{n, \alpha}=-\frac{4^{\alpha} \pi^{-n / 2} \Gamma\left(\frac{n+2 \alpha}{2}\right)}{\Gamma(-\alpha)}>0 .
$$

A function $u \in C(H)$ is said to be $L^{(\alpha)}$-harmonic on $H$ if $u$ satisfies the following condition: for every $\psi \in C_{c}^{\infty}(H)$,

$$
\int_{H}\left|u \cdot \tilde{L}^{(\alpha)} \psi\right| d V<\infty \quad \text { and } \quad \int_{H} u \cdot \tilde{L}^{(\alpha)} \psi d V=0
$$

where $\tilde{L}^{(\alpha)}=-\partial_{t}+\left(-\Delta_{x}\right)^{\alpha}$ is the adjoint operator of $L^{(\alpha)}$. By $(2.1)$ and the compactness of $\operatorname{supp}(\psi)$ (the support of $\psi$ ), there exist $0<t_{1}<t_{2}<\infty$ and 
a constant $C>0$ such that $\operatorname{supp}\left(\tilde{L}^{(\alpha)} \psi\right) \subset S=\mathbb{R}^{n} \times\left[t_{1}, t_{2}\right]$ and $\left|\tilde{L}^{(\alpha)} \psi(x, t)\right| \leq$ $C(1+|x|)^{-n-2 \alpha}$ for all $(x, t) \in S$. Thus, the integrability condition of $(2.2)$ is equivalent to the following: for any $0<t_{1}<t_{2}<\infty$,

$$
\int_{t_{1}}^{t_{2}} \int_{\mathbb{R}^{n}}|u(x, t)|(1+|x|)^{-n-2 \alpha} d V(x, t)<\infty .
$$

We introduce the fundamental solution of $L^{(\alpha)}$. For $x \in \mathbb{R}^{n}$, the fundamental solution $W^{(\alpha)}$ of $L^{(\alpha)}$ is defined by

$$
W^{(\alpha)}(x, t)= \begin{cases}\frac{1}{(2 \pi)^{n}} \int_{\mathbb{R}^{n}} \exp \left(-t|\xi|^{2 \alpha}+\sqrt{-1} x \cdot \xi\right) d \xi, & t>0 \\ 0, & t \leq 0\end{cases}
$$

where $x \cdot \xi$ denotes the usual inner product on $\mathbb{R}^{n}$. It is known that $W^{(\alpha)}$ is $L^{(\alpha)}$-harmonic on $H$ and $W^{(\alpha)} \in C^{\infty}(H)$.

We define fractional integral and differential operators. Let $C\left(\mathbb{R}_{+}\right)$be the set of all continuous functions on $\mathbb{R}_{+}=(0, \infty)$. For a positive real number $\kappa$, let $\mathscr{F} \mathscr{C}^{-\kappa}$ be the set of all functions $\varphi \in C\left(\mathbb{R}_{+}\right)$such that there exists a constant $\varepsilon>0$ with $\varphi(t)=O\left(t^{-\kappa-\varepsilon}\right)$ as $t \rightarrow \infty$. We remark that $\mathscr{F} \mathscr{C}^{-v} \subset \mathscr{F} \mathscr{C}^{-\kappa}$ if $0<$ $\kappa \leq v$. For $\varphi \in \mathscr{F} \mathscr{C}^{-\kappa}$, we can define the fractional integral of $\varphi$ with order $\kappa$ by

$$
\mathscr{D}_{t}^{-\kappa} \varphi(t)=\frac{1}{\Gamma(\kappa)} \int_{0}^{\infty} \tau^{\kappa-1} \varphi(t+\tau) d \tau, \quad t \in \mathbb{R}_{+} .
$$

Furthermore, let $\mathscr{F} \mathscr{C}^{\kappa}$ be the set of all functions $\varphi \in C^{\lceil\kappa]}\left(\mathbb{R}_{+}\right)$such that $\partial_{t}^{\lceil\kappa\rceil} \varphi \in$ $\mathscr{F} \mathscr{C}^{-(\lceil\kappa\rceil-\kappa)}$, where $\lceil\kappa\rceil$ is the smallest integer greater than or equal to $\kappa$. Also, we define $\mathscr{F} \mathscr{C}^{0}=C\left(\mathbb{R}_{+}\right)$. For $\varphi \in \mathscr{F} \mathscr{C}^{\kappa}$, we can also define the fractional derivative of $\varphi$ with order $\kappa$ by

$$
\mathscr{D}_{t}^{\kappa} \varphi(t)=\mathscr{D}_{t}^{-(\lceil\kappa\rceil-\kappa)}\left(-\partial_{t}\right)^{\lceil\kappa\rceil} \varphi(t), \quad t \in \mathbb{R}_{+},
$$

where we define $\mathscr{D}_{t}^{0} \varphi=\varphi$.

Now, we describe basic properties of fractional derivatives of the fundamental solution $W^{(\alpha)}$, which were given in [1]. Let $\mathbb{N}_{0}=\mathbb{N} \cup\{0\}$. For a multi-index $\beta=\left(\beta_{1}, \ldots, \beta_{n}\right) \in \mathbb{N}_{0}^{n}$, let $\partial_{x}^{\beta}=\partial^{|\beta|} / \partial x_{1}^{\beta_{1}} \cdots \partial x_{n}^{\beta_{n}}$, where $|\beta|=$ $\beta_{1}+\cdots+\beta_{n}$.

Lemma 1 (Theorem 3.1 of [1]). Let $0<\alpha \leq 1, \beta \in \mathbb{N}_{0}^{n}$, and $\kappa>-\frac{n}{2 \alpha}$. Then, the following statements hold.

(1) The both derivatives $\partial_{x}^{\beta} \mathscr{D}_{t}^{\kappa} W^{(\alpha)}(x, t)$ and $\mathscr{D}_{t}^{\kappa} \partial_{x}^{\beta} W^{(\alpha)}(x, t)$ are welldefined and $\partial_{x}^{\beta} \mathscr{D}_{t}^{\kappa} W^{(\alpha)}(x, t)=\mathscr{D}_{t}^{\kappa} \partial_{x}^{\beta} W^{(\alpha)}(x, t)$. Moreover, there exists a con- 
stant $C>0$ such that

$$
\left|\partial_{x}^{\beta} \mathscr{D}_{t}^{\kappa} W^{(\alpha)}(x, t)\right| \leq C\left(t+|x|^{2 \alpha}\right)^{-(n+|\beta|) /(2 \alpha)-\kappa}
$$

for all $(x, t) \in H$.

(2) Let $v$ be a real number such that $\kappa+v>-\frac{n}{2 \alpha}$. Then,

$$
\mathscr{D}_{t}^{v} \partial_{x}^{\beta} \mathscr{D}_{t}^{\kappa} W^{(\alpha)}(x, t)=\partial_{x}^{\beta} \mathscr{D}_{t}^{\kappa+v} W^{(\alpha)}(x, t)
$$

for all $(x, t) \in H$.

(3) $\partial_{x}^{\beta} \mathscr{D}_{t}^{\kappa} W^{(\alpha)}$ is $L^{(\alpha)}$-harmonic on $H$.

We present basic properties of the function $\omega_{\alpha}^{v}$ defined in Theorem 1 .

Lemma 2 (Lemma 5.6 of [2]). Let $0<\alpha \leq 1, \sigma>-m(\alpha)$, and $v>-\frac{n}{2 \alpha}$. If $\rho>-1$ and $\eta:=v-\rho-1>-m(\alpha)$, then there exists a constant $C=$ $C(n, \alpha, v, \rho)>0$ such that

$$
\int_{H}\left|\omega_{\alpha}^{v}(x, t ; y, s)\right| s^{\rho} d V(y, s) \leq C F_{\alpha, \eta}(x, t)
$$

for all $(x, t) \in H$, where

$$
F_{\alpha, \eta}(x, t):= \begin{cases}1+|x|^{-2 \alpha \eta}+t^{-\eta}, & 0>\eta>-m(\alpha), \\ 1+\log (1+|x|)+|\log t|, & \eta=0, \\ 1+t^{-\eta}, & \eta>0 .\end{cases}
$$

Lemma 3 (Lemma 5 of [7]). Let $\theta, c \in \mathbb{R}$. If $\theta>-1$ and $\theta-c+\frac{n}{2 \alpha}+1<$ 0 , then there exists a constant $C=C(n, \alpha, \theta, c)>0$ such that

$$
\int_{H} \frac{s^{\theta}}{\left(t+s+|x-y|^{2 \alpha}\right)^{c}} d V(y, s)=C t^{\theta-c+n /(2 \alpha)+1}
$$

for all $(x, t) \in H$.

\section{A generalization of reproducing formula for parabolic Bloch functions}

In this section, we give a generalization of the reproducing formula on parabolic Bloch spaces. First, we present basic properties of parabolic Bloch functions.

Lemma 4 (Theorem 3.2 and Proposition 5.4 of [2]). Let $0<\alpha \leq 1, \sigma>$ $-m(\alpha), \gamma \in \mathbb{N}_{0}^{n}$, and $\kappa$ be a real number such that $\kappa=0$ or $\kappa>\max \{0,-\sigma\}$. If $u \in \mathscr{B}_{\alpha}(\sigma)$, then the following statements hold. 
(1) There exists a constant $C>0$ independent of $u$ such that

$$
|u(x, t)| \leq C\|u\|_{\mathscr{B}_{\alpha}(\sigma)} F_{\alpha, \sigma}(x, t)
$$

for all $(x, t) \in H$, where $F_{\alpha, \sigma}(x, t)$ is the function defined in Lemma 2.

(2) The derivatives $\partial_{x}^{\gamma} \mathscr{D}_{t}^{\kappa} u(x, t)$ and $\mathscr{D}_{t}^{\kappa} \partial_{x}^{\gamma} u(x, t)$ can be defined, and the equation $\partial_{x}^{\gamma} \mathscr{D}_{t}^{\kappa} u(x, t)=\mathscr{D}_{t}^{\kappa} \partial_{x}^{\gamma} u(x, t)$ holds. Furthermore, if $(\gamma, \kappa) \neq(0,0)$, then there exists a constant $C=C(n, \alpha, \sigma, \gamma, \kappa)>0$ such that

$$
\left|\partial_{x}^{\gamma} \mathscr{D}_{t}^{\kappa} u(x, t)\right| \leq C t^{-(|\gamma| /(2 \alpha)+\kappa+\sigma)}\|u\|_{\mathscr{B}_{\alpha}(\sigma)}
$$

for all $(x, t) \in H$.

(3) $\partial_{x}^{\gamma} \mathscr{D}_{t}^{\kappa} u$ is $L^{(\alpha)}$-harmonic on $H$.

The following lemma is the reproducing formula on parabolic Bloch spaces.

Lemma 5 (Theorem 5.7 of [2]). Let $0<\alpha \leq 1$ and $\sigma>-m(\alpha)$. If real numbers $\kappa$ and $v$ satisfy $\kappa>\max \{0,-\sigma\}$ and $v>\sigma$, then the reproducing formula

$$
u(x, t)=\frac{2^{\kappa+v}}{\Gamma(\kappa+v)} \int_{H} \mathscr{D}_{t}^{\kappa} u(y, s) \omega_{\alpha}^{v}(x, t ; y, s) s^{\kappa+v-1} d V(y, s)
$$

holds for all $u \in \tilde{\mathscr{B}}_{\alpha}(\sigma)$ and $(x, t) \in H$. If $\kappa=0$ and $v>\max \{0, \sigma\}$, then (3.1) also holds.

Lemma 6 (Theorem 3 of [4]). Let $0<\alpha \leq 1$ and $\sigma_{1}, \sigma_{2}>-m(\alpha)$. Then, $\tilde{\mathscr{B}}_{\alpha}\left(\sigma_{1}\right) \cong \tilde{\mathscr{B}}_{\alpha}\left(\sigma_{2}\right)$ under the relation $\mathscr{D}_{t}^{-\sigma_{1}+\kappa} u=\mathscr{D}_{t}^{-\sigma_{2}+\kappa} v$ for $u \in \tilde{\mathscr{B}}_{\alpha}\left(\sigma_{1}\right)$ and $v \in \tilde{\mathscr{B}}_{\alpha}\left(\sigma_{2}\right)$, where $\kappa>\max \left\{0, \sigma_{1}, \sigma_{2}\right\}$. Moreover, there exists a constant $C>0$ such that

$$
C^{-1}\|v\|_{\mathscr{B}_{\alpha}\left(\sigma_{2}\right)} \leq\|u\|_{\mathscr{B}_{\alpha}\left(\sigma_{1}\right)} \leq C\|v\|_{\mathscr{B}_{\alpha}\left(\sigma_{2}\right)}
$$

Next, we introduce the following integral operator induced by $\omega_{\alpha}^{v}$. Let $0<\alpha \leq 1$ and $v, \rho$ be real numbers. For a function $f$ on $H$, the integral operator $\Pi_{\alpha}^{v, \rho}$ is defined by

$$
\Pi_{\alpha}^{v, \rho} f(x, t):=\int_{H} f(y, s) \omega_{\alpha}^{v}(x, t ; y, s) s^{\rho} d V(y, s) .
$$

We give the following two lemmas, which give some properties with respect to the operator $\Pi_{\alpha}^{v, \rho}$. We denote by $L^{\infty}(H)$ the set of all essentially bounded Lebesgue measurable functions on $H$, and $\|f\|_{L^{\infty}(H)}=\operatorname{esssup}\{|f(x, t)| ;(x, t) \in$ $H\}$.

Lemma 7 (Theorem 5.8 of [2]). Let $0<\alpha \leq 1, \sigma>-m(\alpha)$, and $v>\sigma$. Then, $\Pi_{\alpha}^{v, v-\sigma-1}$ is a bounded operator from $L^{\infty}(H)$ onto $\tilde{\mathscr{B}}_{\alpha}(\sigma)$. 
Lemma 8. Let $0<\alpha \leq 1$ and $\rho>-1$. Also, let $\kappa>0$ and $v>-\frac{n}{2 \alpha}$ such that $v-\rho-1>-m(\alpha)$. Then,

$$
\mathscr{D}_{t}^{\kappa} \Pi_{\alpha}^{v, \rho} f(x, t)=\int_{H} f(y, s) \mathscr{D}_{t}^{\kappa+v} W^{(\alpha)}(x-y, t+s) s^{\rho} d V(y, s)
$$

holds for all $f \in L^{\infty}(H)$ and $(x, t) \in H$.

Proof. By (1) of Lemma 1, Lemma 2, and Lemma 3, a differentiation under the integral sign and the Fubini theorem show the assertion, directly.

In order to show Theorem 1, we prepare the following lemma, which is a generalization of Lemma 5 .

Lemma 9. Let $0<\alpha \leq 1$ and $\sigma, \rho>-m(\alpha)$. Also, let $\kappa>\max \{0,-\sigma\}$ and $v>\rho$. Then, for any $u \in \tilde{\mathscr{B}}_{\alpha}(\sigma)$, the following statements hold:

(1) If $\rho=\sigma$, then

$$
2^{\kappa+v} \Pi_{\alpha}^{v, v-\rho-1}\left(s^{\kappa+\sigma} \mathscr{D}_{t}^{\kappa} u\right)(x, t)=\Gamma(\kappa+v) u(x, t)
$$

holds for all $(x, t) \in H$.

(2) If $\rho<\sigma$, then there exists $v \in \tilde{\mathscr{B}}_{\alpha}(\rho)$ such that

$$
\mathscr{D}_{t}^{\kappa} u=2^{\sigma-\rho} \mathscr{D}_{t}^{\kappa+\sigma-\rho} v
$$

on $H$ and

$$
2^{\kappa+v} \Pi_{\alpha}^{v, v-\rho-1}\left(s^{\kappa+\sigma} \mathscr{D}_{t}^{\kappa} u\right)(x, t)=\Gamma(\kappa+v+\sigma-\rho) v(x, t)
$$

holds for all $(x, t) \in H$.

(3) If $\rho>\max \{0, \sigma\}$, then $v(x, t):=2^{\rho-\sigma}\left(\mathscr{D}_{t}^{\rho-\sigma} u(x, t)-\mathscr{D}_{t}^{\rho-\sigma} u(0,1)\right) \in$ $\tilde{\mathscr{B}}_{\alpha}(\rho)$ and

$$
2^{\kappa+v} \Pi_{\alpha}^{v, v-\rho-1}\left(s^{\kappa+\sigma} \mathscr{D}_{t}^{\kappa} u\right)(x, t)=\Gamma(\kappa+v+\sigma-\rho) v(x, t)
$$

holds for all $(x, t) \in H$.

Proof. We note that the function $t^{\kappa+\sigma} \mathscr{D}_{t}^{\kappa} u$ is in $L^{\infty}(H)$ by (2) of Lemma 4.

(1) The assertion is the same as that of Lemma 5.

(2) By Lemma 6, there exists $v \in \tilde{\mathscr{B}}_{\alpha}(\rho)$ such that $\mathscr{D}_{t}{ }_{t} u=2^{\sigma-\rho} \mathscr{D}_{t}^{\kappa+\sigma-\rho} v$ on $H$. Since $\kappa+\sigma-\rho>\max \{0,-\rho\}$ and $v>\rho$, Lemma 5 implies that

$$
\begin{aligned}
2^{\kappa+v} & \Pi_{\alpha}^{v, v-\rho-1}\left(s^{\kappa+\sigma} \mathscr{D}_{t}^{\kappa} u\right)(x, t) \\
& =2^{\kappa+v+\sigma-\rho} \int_{H} \mathscr{D}_{t}^{\kappa+\sigma-\rho} v(y, s) \omega_{\alpha}^{v}(x, t ; y, s) s^{\kappa+v+\sigma-\rho-1} d V(y, s) \\
& =\Gamma(\kappa+v+\sigma-\rho) v(x, t) .
\end{aligned}
$$


(3) By Lemmas 5 and 8, we have

$$
\begin{aligned}
\mathscr{D}_{t}^{\rho-\sigma} u(x, t)= & \frac{2^{\kappa+v+\sigma-\rho}}{\Gamma(\kappa+v+\sigma-\rho)} \mathscr{D}_{t}^{\rho-\sigma} \Pi_{\alpha}^{v+\sigma-\rho, v-\rho-1}\left(s^{\kappa+\sigma} \mathscr{D}_{t}^{\kappa} u\right)(x, t) \\
= & \frac{2^{\kappa+v+\sigma-\rho}}{\Gamma(\kappa+v+\sigma-\rho)} \int_{H} \mathscr{D}_{t}^{\kappa} u(y, s) \mathscr{D}_{t}^{v} W^{(\alpha)} \\
& \times(x-y, t+s) s^{\kappa+v+\sigma-\rho-1} d V(y, s) .
\end{aligned}
$$

Put $v(x, t)=2^{\rho-\sigma}\left(\mathscr{D}_{t}^{\rho-\sigma} u(x, t)-\mathscr{D}_{t}^{\rho-\sigma} u(0,1)\right)$. Since $\rho-\sigma>\max \{0,-\sigma\}$, (2) and (3) of Lemma 4 imply that $v \in \tilde{\mathscr{B}}_{\alpha}(\rho)$. Moreover by (3.3), we obtain

$$
\Gamma(\kappa+v+\sigma-\rho) v(x, t)=2^{\kappa+v} \Pi_{\alpha}^{v, v-\rho-1}\left(s^{\kappa+\sigma} \mathscr{D}_{t}^{\kappa} u\right)(x, t)
$$

for all $(x, t) \in H$.

\section{The reproducing formula on $\mathscr{B}_{\alpha}(\sigma, \rho)$}

In this section, we show several properties of $\mathscr{B}_{\alpha}(\sigma, \rho)$-functions. We recall the definition of a fractional differential operator $\mathscr{E}_{t}^{\kappa}$. Let $\kappa$ be a real number. Also, let $u_{0}$ and $u_{1}$ be functions on $H$ and $u(x, t)=u_{0}(x, t)+$ $t^{\rho} u_{1}(x, t)$. Then, we define

$$
\mathscr{E}_{t}{ }_{t} u(x, t)=\mathscr{D}_{t}^{\kappa} u_{0}(x, t)+t^{\rho} \mathscr{D}_{t}^{\kappa} u_{1}(x, t)
$$

THEOREM 3. Let $0<\alpha \leq 1, \sigma>-m(\alpha)$, and $\rho \neq 0$ be real numbers such that $\sigma+\rho>-m(\alpha)$. Also, let $\gamma \in \mathbb{N}_{0}^{n}$ and $\kappa>\max \{0,-\sigma,-\sigma-\rho\}$. For $u \in$ $\mathscr{B}_{\alpha}(\sigma, \rho)$, the following statements hold.

(1) There exists a constant $C>0$ independent of $u$ such that

$$
|u(x, t)| \leq C\left(F_{\alpha, \sigma}(x, t)+t^{\rho} F_{\alpha, \sigma+\rho}(x, t)\right)\|u\|_{\mathscr{B}_{\alpha}(\sigma, \rho)}
$$

for all $(x, t) \in H$, where $F_{\alpha, \sigma}(x, t)$ is the function defined in Lemma 2.

(2) The derivative $\partial_{x}^{\gamma} \mathscr{E}_{t}{ }_{t} u$ is well-defined. Moreover, there exists a constant $C>0$ independent of $u$ such that

$$
\left|\partial_{x}^{\gamma} \mathscr{E}_{t}^{\kappa} u(x, t)\right| \leq C t^{-(|\gamma| /(2 \alpha)+\kappa+\sigma)}\|u\|_{\mathscr{B}_{\alpha}(\sigma, \rho)}
$$

for all $(x, t) \in H$.

Proof. Let $u \in \mathscr{B}_{\alpha}(\sigma, \rho)$. Then, we can take $u_{0} \in \tilde{\mathscr{B}}_{\alpha}(\sigma)$ and $u_{1} \in$ $\tilde{\mathscr{B}}_{\alpha}(\sigma+\rho)$ such that $u(x, t)=u_{0}(x, t)+t^{\rho} u_{1}(x, t)$.

(1) The assertion is shown by (1) of Lemma 4, directly. 
(2) By (2) of Lemma 4, the derivative $\partial_{x}^{\gamma} \mathscr{E}_{t}^{\kappa} u$ is well-defined, and there exists a constant $C>0$ such that

$$
\left|\partial_{x}^{\gamma} \mathscr{E}_{t}^{\kappa} u(x, t)\right| \leq\left|\partial_{x}^{\gamma} \mathscr{D}_{t}^{\kappa} u_{0}(x, t)\right|+t^{\rho}\left|\partial_{x}^{\gamma} \mathscr{D}_{t}^{\kappa} u_{1}(x, t)\right| \leq C t^{-(|\gamma| /(2 \alpha)+\kappa+\sigma)}\|u\|_{\mathscr{B}_{\alpha}(\sigma, \rho)} .
$$

This completes the proof.

Now, we show Theorem 1.

Proof of Theorem 1. Let $u \in \mathscr{B}_{\alpha}(\sigma, \rho)$. Then, we can take $u_{0} \in \tilde{\mathscr{B}}_{\alpha}(\sigma)$ and $u_{1} \in \tilde{\mathscr{B}}_{\alpha}(\sigma+\rho)$ such that $u=u_{0}+t^{\rho} u_{1}$ on $H$. By Lemma 2 and the proof of Theorem 3 (2), the right-hand side of (1.1) is well-defined. Hence, we obtain

$$
\begin{aligned}
2^{v+\kappa} \int_{H} \mathscr{E}_{t}^{\kappa} u(y, s) \mathscr{R}_{\alpha}^{v, \kappa, \rho}(x, t ; y, s) s^{v+\kappa-1} d V(y, s) \\
=\sum_{k, \ell, j=0}^{1} C_{k, \ell} 2^{v+\kappa+(k+\ell) \rho} t^{k \rho} \\
\quad \times \int_{H} s^{j \rho} \mathscr{D}_{t}^{\kappa} u_{j}(y, s) \omega_{\alpha}^{v+(k+\ell) \rho}(x, t ; y, s) s^{\kappa+v+\ell \rho-1} d V(y, s) \\
=\sum_{k, \ell, j=0}^{1} C_{k, \ell} 2^{v+\kappa+(k+\ell) \rho} t^{k \rho}\left(\Pi_{\alpha}^{v+(k+\ell) \rho, v+\ell \rho-\sigma-1}\left(s^{\kappa+\sigma+j \rho} \mathscr{D}_{t}^{\kappa} u_{j}\right)(x, t)\right) .
\end{aligned}
$$

When $(k, j)=(0,0)$ and $(1,1),(1)$ of Lemma 9 implies that

$$
C_{0, \ell} 2^{v+\kappa+\ell \rho} \Pi_{\alpha}^{v+\ell \rho, v+\ell \rho-\sigma-1}\left(s^{\kappa+\sigma} \mathscr{D}_{t}^{\kappa} u_{0}\right)(x, t)=C_{0, \ell} \Gamma(v+\kappa+\ell \rho) u_{0}(x, t)
$$

and

$$
\begin{gathered}
C_{1, \ell} t^{\rho} 2^{v+\kappa+(\ell+1) \rho} \Pi_{\alpha}^{v+(\ell+1) \rho, v+(\ell+1) \rho-(\sigma+\rho)-1}\left(s^{\kappa+\sigma+\rho} \mathscr{D}_{t}^{\kappa} u_{1}\right)(x, t) \\
\quad=C_{1, \ell} \Gamma(v+\kappa+(\ell+1) \rho) t^{\rho} u_{1}(x, t)
\end{gathered}
$$

hold for all $\ell=0,1$. Let $(k, j)=(1,0)$. Then by (3) of Lemma 9, there exists $v_{0} \in \tilde{\mathscr{B}}_{\alpha}(\sigma+\rho)$ such that

$$
\begin{gathered}
C_{1, \ell} 2^{v+\kappa+(\ell+1) \rho} t^{\rho} \Pi_{\alpha}^{v+(\ell+1) \rho, v+(\ell+1) \rho-(\sigma+\rho)-1}\left(s^{\kappa+\sigma} \mathscr{D}_{t}^{\kappa} u_{0}\right)(x, t) \\
=C_{1, \ell} \Gamma(v+\kappa+\ell \rho) t^{\rho} v_{0}(x, t)
\end{gathered}
$$

for all $\ell=0,1$, because $\rho>0$ and $\sigma+\rho>0$. Let $(k, j)=(0,1)$. Then by (2) of Lemma 9, there exists $v_{1} \in \tilde{\mathscr{B}}_{\alpha}(\sigma)$ such that 


$$
\begin{gathered}
C_{0, \ell} 2^{v+\kappa+\ell \rho} \Pi_{\alpha}^{v+\ell \rho, v+\ell \rho-\sigma-1}\left(s^{\kappa+\sigma+\rho} \mathscr{D}_{t}^{\kappa} u_{1}\right)(x, t) \\
=C_{0, \ell} \Gamma(v+\kappa+(\ell+1) \rho) v_{1}(x, t)
\end{gathered}
$$

for all $\ell=0,1$.

Since the constants $C_{0,0}, C_{0,1}, C_{1,0}, C_{1,1}$ satisfy the condition (1.2), we can get the desired result.

REMARK 1. Since $v+\kappa>0$ and $\rho>0$, the matrix

$$
\left(\begin{array}{cc}
\Gamma(v+\kappa) & \Gamma(v+\kappa+\rho) \\
\Gamma(v+\kappa+\rho) & \Gamma(v+\kappa+2 \rho)
\end{array}\right)
$$

is invertible. Because the function $\log \Gamma(x)$ is strictly convex on $\mathbb{R}_{+}=(0, \infty)$. Hence, the constants $C_{0,0}, C_{0,1}, C_{1,0}, C_{1,1}$ in Theorem 1 can be written as follows:

$$
\begin{aligned}
& C_{0,0}=\frac{\Gamma(v+\kappa+2 \rho)}{\Gamma(v+\kappa) \Gamma(v+\kappa+2 \rho)-(\Gamma(v+\kappa+\rho))^{2}}, \\
& C_{0,1}=C_{1,0}=\frac{\Gamma(v+\kappa+\rho)}{(\Gamma(v+\kappa+\rho))^{2}-\Gamma(v+\kappa) \Gamma(v+\kappa+2 \rho)}, \\
& C_{1,1}=\frac{\Gamma(v+\kappa)}{\Gamma(v+\kappa) \Gamma(v+\kappa+2 \rho)-(\Gamma(v+\kappa+\rho))^{2}} .
\end{aligned}
$$

Finally, we introduce an integral operator induced by the kernel function $\mathscr{R}_{\alpha}^{v, \kappa, \rho}$. Let $\kappa, v, \sigma$, and $\rho$ be real numbers. For a function $f$ on $H$, an integral operator $P_{\alpha}^{v, \kappa,(\sigma, \rho)}$ is defined by

$$
P_{\alpha}^{v, \kappa,(\sigma, \rho)} f(x, t)=\int_{H} f(y, s) \mathscr{R}_{\alpha}^{v, \kappa, \rho}(x, t ; y, s) s^{v-\sigma-1} d V(y, s) .
$$
$P_{\alpha}^{v, \kappa,(\sigma, \rho)}$.

THEOREM 4. Let $0<\alpha \leq 1, \sigma>-m(\alpha)$, and $\rho>0$ such that $\sigma+\rho>0$. Also, let $\kappa>\max \{0,-\sigma\}$ and $v>\sigma$. Then, $P_{\alpha}^{v, \kappa,(\sigma, \rho)}$ is a bounded linear operator from $L^{\infty}(H)$ onto $\mathscr{B}_{\alpha}(\sigma, \rho)$.

Proof. We show that $P_{\alpha}^{v, \kappa,(\sigma, \rho)}$ is bounded linear operator from $L^{\infty}(H)$ to $\mathscr{B}_{\alpha}(\sigma, \rho)$. Let $f \in L^{\infty}(H)$. Then, we have

$$
\begin{aligned}
P_{\alpha}^{v, \kappa,(\sigma, \rho)} f(x, t)= & C_{0,0} \Pi_{\alpha}^{v, v-\sigma-1} f(x, t)+C_{0,1} 2^{\rho} \Pi_{\alpha}^{v+\rho, v+\rho-\sigma-1} f(x, t) \\
& +t^{\rho}\left\{C_{1,0} 2^{\rho} \Pi_{\alpha}^{v+\rho, v-\sigma-1} f(x, t)+C_{1,1} 4^{\rho} \Pi_{\alpha}^{v+2 \rho, v+\rho-\sigma-1} f(x, t)\right\},
\end{aligned}
$$


where constants $C_{0,0}, C_{0,1}, C_{1,0}, C_{1,1}$ and the operator $\Pi_{\alpha}^{v, v-\sigma-1}$ are defined as (1.2) and (3.2), respectively. Put

$$
\begin{aligned}
& v_{0}(x, t)=C_{0,0} \Pi_{\alpha}^{v, v-\sigma-1} f(x, t)+C_{0,1} 2^{\rho} \Pi_{\alpha}^{v+\rho, v+\rho-\sigma-1} f(x, t), \\
& v_{1}(x, t)=C_{1,0} 2^{\rho} \Pi_{\alpha}^{v+\rho, v-\sigma-1} f(x, t)+C_{1,1} 4^{\rho} \Pi_{\alpha}^{v+2 \rho, v+\rho-\sigma-1} f(x, t) .
\end{aligned}
$$

Then, Lemma 7 implies that $v_{0} \in \tilde{\mathscr{B}}_{\alpha}(\sigma), v_{1} \in \tilde{\mathscr{B}}_{\alpha}(\sigma+\rho)$, and there exists a constant $C>0$ independent of $f$ such that

$$
\left\|P_{\alpha}^{v, \kappa,(\sigma, \rho)} f(x, t)\right\|_{\mathscr{B}_{\alpha}(\sigma, \rho)}=\left\|v_{0}\right\|_{\mathscr{B}_{\alpha}(\sigma)}+\left\|v_{1}\right\|_{\mathscr{B}_{\alpha}(\sigma+\rho)} \leq C\|f\|_{L^{\infty}(H)} .
$$

Namely, $P_{\alpha}^{v, \kappa,(\sigma, \rho)}$ is a bounded linear operator from $L^{\infty}(H)$ to $\mathscr{B}_{\alpha}(\sigma, \rho)$.

We show that the operator $P_{\alpha}^{v, \kappa,(\sigma, \rho)}$ is surjective. For $u \in \mathscr{B}_{\alpha}(\sigma, \rho)$, put

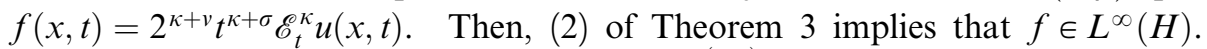
Moreover by Theorem 1, we obtain $u=P_{\alpha}^{v, \kappa,(\sigma, \rho)} f$ on $H$. This completes the proof.

As an application of Theorem 4 and (2) of Theorem 3, we give the estimate of the following normal derivative norm of $\mathscr{B}_{\alpha}(\sigma, \rho)$-functions.

Corollary 1. Let $0<\alpha \leq 1, \sigma>-m(\alpha)$, and $\rho>0$ such that $\sigma+\rho>0$. Also, let $\kappa>\max \{0,-\sigma\}$. Then there exists a constant $C>0$ such that for any $u \in \mathscr{B}_{\alpha}(\sigma, \rho)$,

$$
C^{-1}\|u\|_{\mathscr{B}_{\alpha}(\sigma, \rho)} \leq\left\|t^{\kappa+\sigma} \mathscr{E}_{t}^{\kappa} u\right\|_{L^{\infty}(H)} \leq C\|u\|_{\mathscr{B}_{\alpha}(\sigma, \rho)} .
$$

\section{Introduction to bi-parabolic Bloch spaces}

In this section, we introduce bi-parabolic Bloch spaces, and investigate several properties.

Let $0<\alpha \leq 1$ and $\sigma>-m(\alpha)$. We define $\tilde{\mathscr{B}}_{\alpha}^{2}(\sigma)=\mathscr{B}_{\alpha}(\sigma, 1)$. In fact, an elementary calculation shows that for any $u \in \mathscr{B}_{\alpha}(\sigma, 1)$, the equation $\left(L^{(\alpha)}\right)^{2} u=$ 0 holds on $H$ and

$$
\sup _{(x, t) \in H} t^{\sigma}\left\{t^{1 / 2 \alpha}|\nabla u(x, t)|+t\left|\mathscr{E}_{t} u(x, t)\right|\right\}<\infty .
$$

We also define the norm on $\tilde{\mathscr{B}}_{\alpha}^{2}(\sigma)$ by

$$
\|u\|_{\tilde{\mathscr{B}}_{\alpha}^{2}(\sigma)}:=\|u\|_{\mathscr{B}_{\alpha}(\sigma, 1)} .
$$

We discuss a dual space of the bi-parabolic Bergman space $\boldsymbol{b}_{\alpha}^{2,1}(\lambda)$. We present previous results with respect to bi-parabolic Bergman spaces. Let $d V^{\lambda}(x, t)=t^{\lambda} d V(x, t)$ on $H$. We denote by $L^{p}\left(H, d V^{\lambda}\right)$ the set of all 
Lebesgue measurable functions $f$ on $H$ which satisfy

$$
\|f\|_{L^{p}(\lambda)}:=\left(\int_{H}|f(x, t)|^{p} d V^{\lambda}(x, t)\right)^{1 / p}<\infty .
$$

Definition 2 (Section 1 of [6]). Let $0<\alpha \leq 1,1 \leq p<\infty$, and $\lambda>-1$. The bi-parabolic Bergman space $\boldsymbol{b}_{\alpha}^{2, p}(\lambda)$ is defined by

$$
\begin{gathered}
\boldsymbol{b}_{\alpha}^{2, p}(\lambda):=\left\{u \in C^{\infty}(H) ;\left(L^{(\alpha)}\right)^{2} u=0, u \in L^{p}\left(H, d V^{\lambda}\right), L^{(\alpha)} u \in L^{p}\left(H, d V^{\lambda+p}\right),\right. \\
\left.{ }^{\forall} t>0 \text { and }{ }^{\forall}(\beta, j) \in \mathbb{N}_{0}^{n+1}, \sup _{x \in \mathbb{R}^{n}}\left|\partial_{x}^{\beta} \partial_{t}^{j} u(x, t)\right|<\infty\right\} .
\end{gathered}
$$

We present the reproducing formula on $\boldsymbol{b}_{\alpha}^{2, p}(\lambda)$.

Lemma 10 (Theorem 4 of [6]). Let $0<\alpha \leq 1,1 \leq p<\infty$, and $\lambda>-1$. Also, let $\kappa>-\frac{\lambda+1}{p}$ and $v>\frac{\lambda+1}{p}$. Then, the reproducing formula

$$
u(x, t)=2^{v+\kappa} \int_{H} \mathscr{E}_{t}^{\kappa} u(y, s) \mathscr{K}_{\alpha}^{2, v, \kappa}(x, t ; y, s) s^{v+\kappa-1} d V(y, s)
$$

holds for all $u \in \boldsymbol{b}_{\alpha}^{2, p}(\lambda)$ and $(x, t) \in H$, where

$$
\begin{aligned}
\mathscr{K}_{\alpha}^{2, v, \kappa}(x, t ; y, s) & \\
= & \frac{v+\kappa+1}{\Gamma(v+\kappa)} \mathscr{D}_{t}^{v} W^{(\alpha)}(x-y, t+s)-\frac{2 s}{\Gamma(v+\kappa)} \mathscr{D}_{t}^{v+1} W^{(\alpha)}(x-y, t+s) \\
& \quad-\frac{2 t}{\Gamma(v+\kappa)} \mathscr{D}_{t}^{v+1} W^{(\alpha)}(x-y, t+s)+\frac{4 t s}{\Gamma(v+\kappa+1)} \mathscr{D}_{t}^{v+2} W^{(\alpha)}(x-y, t+s) .
\end{aligned}
$$

Moreover, the assertion also holds when $p=1$ and $v=\lambda+1$.

Here, we remark that the kernel function $\mathscr{K}_{\alpha}^{2, v, \kappa}(\cdot, \cdot ; \cdot, \cdot)$ is symmetric on $H \times H$, that is, $\mathscr{K}_{\alpha}^{2, v, \kappa}(x, t ; y, s)=\mathscr{K}_{\alpha}^{2, v, \kappa}(y, s ; x, t)$ for all $(x, t),(y, s) \in H$.

Now, we show Theorem 2.

Proof of Theorem 2. For $v \in \tilde{\mathscr{B}}_{\alpha}^{2}(\sigma)$, we define $l(v)=\Phi_{v}$. Then, it is easy to see that $\imath: \tilde{\mathscr{B}}_{\alpha}^{2}(\sigma) \rightarrow\left(\boldsymbol{b}_{\alpha}^{2,1}(\lambda)\right)^{*}$ and $\left\|\Phi_{v}\right\| \leq C\|v\|_{\tilde{\mathscr{B}}_{\alpha}^{2}(\sigma)}$. In fact, (2) of Theorem 3 shows that

$$
\left|\Phi_{v}(u)\right| \leq C\|u\|_{L^{1}(\lambda)}\|v\|_{\tilde{\mathscr{B}}_{\alpha}^{2}(\sigma)}
$$

for all $u \in \boldsymbol{b}_{\alpha}^{2,1}(\lambda)$.

We show that the mapping $\imath$ is injective. Let $v \in \tilde{\mathscr{B}}_{\alpha}^{2}(\sigma)$ such that $\imath(v)=0$. Then by Theorem 1, we have 


$$
\begin{aligned}
v(x, t) & =2^{\lambda+\sigma+2} \int_{H} \mathscr{E}_{t} v(y, s) \mathscr{R}_{\alpha}^{\lambda+\sigma+1,1,1}(x, t ; y, s) s^{\lambda+\sigma+1} d V(y, s) \\
& =\Phi_{v}\left(\mathscr{R}_{\alpha}^{\lambda+\sigma+1,1,1}(x, t ; \cdot, \cdot)\right) .
\end{aligned}
$$

Here, we have $\mathscr{R}_{\alpha}^{\lambda+\sigma+1,1,1}(x, t ; \cdot, \cdot) \in \boldsymbol{b}_{\alpha}^{2,1}(\lambda)$ by (1), (2), (3) of Lemma 1 and Lemma 2. Therefore, we obtain $v=0$.

We show that the mapping $l$ is surjective. Let $\Phi \in\left(\boldsymbol{b}_{\alpha}^{2,1}(\lambda)\right)^{*}$. Since $\boldsymbol{b}_{\alpha}^{2,1}(\lambda) \subset L^{1}\left(H, d V^{\lambda}\right)$, the Hahn-Banach theorem and the Riesz representation theorem show that there exists a function $f \in L^{\infty}(H)$ with

$$
\Phi(u)=\int_{H} u(x, t) f(x, t) t^{\lambda} d V(x, t)
$$

for all $u \in \boldsymbol{b}_{\alpha}^{2,1}(\lambda)$ and $\|f\|_{L^{\infty}(H)}=\|\Phi\|$. Put $v(x, t)=P_{\alpha}^{\lambda+\sigma+1,1,(\sigma, 1)} f(x, t)$. Then by Theorem $4, v \in \tilde{\mathscr{B}}_{\alpha}^{2}(\sigma)$ and there exists a constant $C>0$ independent of $v$ such that $\|v\|_{\tilde{\mathscr{B}}_{\alpha}^{2}(\sigma)} \leq C\|\Phi\|$. We claim $\Phi(u)=\langle u, v\rangle_{\lambda, \sigma}$. In fact, we have

$$
\langle u, v\rangle_{\lambda, \sigma}=2^{\lambda+\sigma+2} \int_{H} u(x, t) \mathscr{E}_{t}\left(P_{\alpha}^{\lambda+\sigma+1,1,(\sigma, 1)} f(x, t)\right) t^{\lambda+\sigma+1} d V(x, t) .
$$

A differentiation under the integral sign shows that

$$
\mathscr{E}_{t}\left(P_{\alpha}^{\lambda+\sigma+1,1,(\sigma, 1)} f(x, t)\right)=\int_{H} f(y, s) \mathscr{K}_{\alpha}^{2, \lambda+\sigma+2,0}(x, t ; y, s) s^{\lambda} d V(y, s)
$$

Since $\mathscr{K}_{\alpha}^{2, \lambda+\sigma+2,0}(\cdot, \cdot ; \cdot, \cdot)$ is symmetric on $H \times H$, the Fubini theorem and Lemma 10 imply that

$$
\langle u, v\rangle_{\lambda, \sigma}=\int_{H} f(y, s) u(y, s) s^{\lambda} d V(y, s)=\Phi(u) .
$$

This completes the proof.

Finally, we discuss a pre-dual space of $\boldsymbol{b}_{\alpha}^{2,1}(\lambda)$. We define bi-parabolic little Bloch spaces $\tilde{\mathscr{B}}_{\alpha, 0}^{2}(\sigma)$. To begin with, we present the definition of parabolic little Bloch spaces in [2]. Let $0<\alpha \leq 1$ and $\sigma>-m(\alpha)$. A function space $\mathscr{B}_{\alpha, 0}(\sigma)$ is defined by the space of functions $u \in \mathscr{B}_{\alpha}(\sigma)$ which satisfy

$$
\lim _{(x, t) \rightarrow \partial H \cup\{\infty\}} t^{\sigma}\left\{t^{1 / 2 \alpha}\left|\nabla_{x} u(x, t)\right|+t\left|\partial_{t} u(x, t)\right|\right\}=0 .
$$

We also denote by $\tilde{\mathscr{B}}_{\alpha, 0}(\sigma)$ the space of functions $u \in \mathscr{B}_{\alpha, 0}(\sigma)$ such that $u(0,1)=0$. We call $\tilde{\mathscr{B}}_{\alpha, 0}(\sigma)\left(\right.$ or $\left.\mathscr{B}_{\alpha, 0}(\sigma)\right)$ the parabolic little Bloch space. We 
note that $\tilde{\mathscr{B}}_{\alpha, 0}(\sigma)$ is a closed subspace of $\tilde{\mathscr{B}}_{\alpha}(\sigma)$. The bi-parabolic little Bloch space $\tilde{\mathscr{B}}_{\alpha, 0}^{2}(\sigma)$ is defined by

$$
\tilde{\mathscr{B}}_{\alpha, 0}^{2}(\sigma):=\left\{u(x, t)=u_{0}(x, t)+t u_{1}(x, t) ; u_{0} \in \tilde{\mathscr{B}}_{\alpha, 0}(\sigma), u_{1} \in \tilde{\mathscr{B}}_{\alpha, 0}(\sigma+1)\right\} .
$$

We note that if $u \in \tilde{\mathscr{B}}_{\alpha, 0}^{2}(\sigma)$, then the following equality holds:

$$
\lim _{(x, t) \rightarrow \partial H \cup\{\infty\}} t^{\sigma}\left\{t^{1 / 2 \alpha}\left|\nabla_{x} u(x, t)\right|+t\left|\mathscr{E}_{t} u(x, t)\right|\right\}=0 .
$$

We claim that a pre-dual space of $\boldsymbol{b}_{\alpha}^{2,1}(\lambda)$ is isomorphic to $\tilde{\mathscr{B}}_{\alpha, 0}^{2}(\sigma)$ under a suitable integral pairing. In order to show our assertion, we prepare the following lemma. Let $C_{0}(H)$ be the set of all continuous functions on $H$ which vanish continuously on $\partial H \cup\{\infty\}$.

LemmA 11. Let $0<\alpha \leq 1$ and $\sigma>-m(\alpha)$. Also, let $\kappa$ and $v$ be real numbers such that $v>\sigma$ and $v+\kappa>0$. Then,

$$
\tilde{\mathscr{B}}_{\alpha, 0}^{2}(\sigma)=\left\{u \in \tilde{\mathscr{B}}_{\alpha}^{2}(\sigma) ; t^{\sigma+1} \mathscr{E}_{t} u \in C_{0}(H)\right\}=\left\{P_{\alpha}^{v, \kappa,(\sigma, 1)} f ; f \in C_{0}(H)\right\}
$$

Proof. By (5.1) and Theorem 1,

$$
\tilde{\mathscr{B}}_{\alpha, 0}^{2}(\sigma) \subseteq\left\{u \in \tilde{\mathscr{B}}_{\alpha}^{2}(\sigma) ; t^{\sigma+1} \mathscr{\mathscr { E }}_{t} u \in C_{0}(H)\right\}
$$

and

$$
\left\{u \in \tilde{\mathscr{B}}_{\alpha}^{2}(\sigma) ; t^{\sigma+1} \mathscr{E}_{t} u \in C_{0}(H)\right\} \subseteq\left\{P_{\alpha}^{v, \kappa,(\sigma, 1)} f ; f \in C_{0}(H)\right\}
$$

are trivial. We show $\left\{P_{\alpha}^{v, \kappa,(\sigma, 1)} f ; f \in C_{0}(H)\right\} \subseteq \tilde{\mathscr{B}}_{\alpha, 0}^{2}(\sigma)$. Let $f \in C_{0}(H)$. In the proof of Theorem 4, we get

$$
\begin{aligned}
P_{\alpha}^{v, \kappa,(\sigma, 1)} f(x, t)= & C_{0,0} \Pi_{\alpha}^{v, v-\sigma-1} f(x, t)+C_{0,1} 2 \Pi_{\alpha}^{v+1, v-\sigma} f(x, t) \\
& +t\left\{C_{1,0} 2 \Pi_{\alpha}^{v+1, v-\sigma-1} f(x, t)+C_{1,1} 4 \Pi_{\alpha}^{v+2, v-\sigma} f(x, t)\right\} \\
= & : v_{0}(x, t)+t v_{1}(x, t) .
\end{aligned}
$$

Here by Lemma 6.2 of [2], we already obtained

$$
\left\{\Pi_{\alpha}^{v, v-\sigma-1} f ; f \in C_{0}(H)\right\}=\tilde{\mathscr{B}}_{\alpha, 0}(\sigma) .
$$

Thus, we have $v_{0} \in \tilde{\mathscr{B}}_{\alpha, 0}(\sigma)$ and $v_{1} \in \tilde{\mathscr{B}}_{\alpha, 0}(\sigma+1)$. Consequently, we have $P_{\alpha}^{v, \kappa,(\sigma, 1)} f \in \tilde{\mathscr{B}}_{\alpha, 0}^{2}(\sigma)$. This completes the proof.

Now, we show the following theorem. 
THEOREM 5. Let $0<\alpha \leq 1, \lambda>-1$, and $\sigma>-m(\alpha)$. Then, $\boldsymbol{b}_{\alpha}^{2,1}(\lambda) \cong$ $\left(\tilde{\mathscr{B}}_{\alpha, 0}^{2}(\sigma)\right)^{*}$ under the pairing

$$
\Phi_{u}(v)=\langle u, v\rangle_{\lambda, \sigma}, \quad v \in \tilde{\mathscr{B}}_{\alpha, 0}^{2}(\sigma),
$$

where $\Phi_{u}$ is the linear functional induced by $u \in \boldsymbol{b}_{\alpha}^{2,1}(\lambda)$. Moreover, there exists a constant $C>0$ independent of $u$ such that

$$
C^{-1}\|u\|_{L^{1}(\lambda)} \leq\left\|\Phi_{u}\right\| \leq C\|u\|_{L^{1}(\lambda)} .
$$

Proof. For $u \in \boldsymbol{b}_{\alpha}^{2,1}(\lambda)$, we define $\imath(u)=\Phi_{u}$. Then, it is easy to see that $l: \boldsymbol{b}_{\alpha}^{2,1}(\lambda) \rightarrow\left(\tilde{\mathscr{B}}_{\alpha, 0}^{2}(\sigma)\right)^{*}$ and $\left\|\Phi_{u}\right\| \leq C\|u\|_{L^{1}(\lambda)}$. In fact, (2) of Theorem 3 shows that

$$
\left|\Phi_{u}(v)\right| \leq C\|u\|_{L^{1}(\lambda)}\|v\|_{\mathscr{B}_{\alpha}(\sigma, 1)}
$$

for all $v \in \tilde{\mathscr{B}}_{\alpha, 0}^{2}(\sigma)$.

We show that the mapping $l$ is injective. Let $u \in \boldsymbol{b}_{\alpha}^{2,1}(\lambda)$ such that $l(u)=$ $\Phi_{u}=0$. Then by Lemma 10 and (2) of Lemma 1, we obtain

$$
\begin{aligned}
u(x, t) & =2^{\lambda+\sigma+2} \int_{H} u(y, s) \mathscr{K}_{\alpha}^{2, \lambda+\sigma+2,0}(x, t ; y, s) s^{\lambda+\sigma+1} d V(y, s) \\
& =\langle u, F(x, t ; \cdot, \cdot)\rangle_{\lambda, \sigma},
\end{aligned}
$$

where

$$
\begin{aligned}
F(x, t ; y, s)= & \frac{\lambda+\sigma+3}{\Gamma(\lambda+\sigma+2)}\left(\mathscr{D}_{t}^{\lambda+\sigma+1} W^{(\alpha)}(x-y, t+s)-\mathscr{D}_{t}^{\lambda+\sigma+1} W^{(\alpha)}(x, t+1)\right) \\
& -\frac{2 t}{\Gamma(\lambda+\sigma+2)}\left(\mathscr{D}_{t}^{\lambda+\sigma+2} W^{(\alpha)}(x-y, t+s)-\mathscr{D}_{t}^{\lambda+\sigma+2} W^{(\alpha)}(x, t+1)\right) \\
& -\frac{2 s}{\Gamma(\lambda+\sigma+2)}\left(\mathscr{D}_{t}^{\lambda+\sigma+2} W^{(\alpha)}(x-y, t+s)-\mathscr{D}_{t}^{\lambda+\sigma+2} W^{(\alpha)}(x, t+1)\right) \\
& +\frac{4 t s}{\Gamma(\lambda+\sigma+3)}\left(\mathscr{D}_{t}^{\lambda+\sigma+3} W^{(\alpha)}(x-y, t+s)-\mathscr{D}_{t}^{\lambda+\sigma+3} W^{(\alpha)}(x, t+1)\right) .
\end{aligned}
$$

By (1) and (3) of Lemma 1, for every $(x, t) \in H$, the function $F(x, t ; \cdot, \cdot)$ belongs to $\tilde{\mathscr{B}}_{\alpha, 0}^{2}(\sigma)$. Hence, we have $u=0$.

We show that the mapping $\imath$ is surjective. Let $\Phi \in\left(\tilde{\mathscr{B}}_{\alpha, 0}^{2}(\sigma)\right)^{*}$. We define a mapping $\Lambda$ on $C_{0}(H)$ by

$$
\Lambda(f)=2^{\lambda+\sigma+2} \Phi\left(P_{\alpha}^{\lambda+\sigma+1,1,(\sigma, 1)} f\right) .
$$

Then, Theorem 4 and Lemma 11 imply that $\Lambda$ is a bounded linear functional on $C_{0}(H)$ and $\|\Lambda\| \leq C\|\Phi\|$. Hence, the Hahn-Banach theorem and the Riesz representation theorem show that there exists a bounded signed measure $\mu$ on 
$H$ which satisfies $\|\mu\|=\|\Lambda\|$ and

$$
\Lambda(f)=\int_{H} f(x, t) d \mu(x, t) .
$$

We define a function $u(y, s)$ such that

$$
u(y, s)=\int_{H} \mathscr{K}_{\alpha}^{2, \lambda+\sigma+2,0}(x, t ; y, s) t^{\sigma+1} d \mu(x, t) .
$$

Then by (1) of Lemma 1 and Lemma 3 , there exists a constant $C>0$ independent of $\mu$ such that

$$
\|u\|_{L^{1}(\lambda)} \leq C \int_{H} \int_{H} \frac{s^{\lambda}}{\left(t+s+|x-y|^{2 \alpha}\right)^{n /(2 \alpha)+\lambda+\sigma+2}} d V(y, s) t^{\sigma+1} d|\mu|(x, t) \leq C\|\mu\|
$$

and

$$
\begin{aligned}
\left\|L^{(\alpha)} u\right\|_{L^{1}(\lambda+1)} & \leq C \int_{H} \int_{H} \frac{s^{\lambda+1}}{\left(t+s+|x-y|^{2 \alpha}\right)^{n /(2 \alpha)+\lambda+\sigma+3}} d V(y, s) t^{\sigma+1} d|\mu|(x, t) \\
& \leq C\|\mu\| .
\end{aligned}
$$

Also, for any $s>0$ and $(\beta, j) \in \mathbb{N}_{0}^{n+1}$, (1) and (2) of Lemma 1 imply that

$$
\sup _{y \in \mathbb{R}}\left|\partial_{x}^{\beta} \partial_{t}^{j} u(y, s)\right|<\infty .
$$

Moreover, it is easy to see that $\left(L^{(\alpha)}\right)^{2} u=0$ on $H$, because for fixed $(x, t) \in H$, $\left(L^{(\alpha)}\right)^{2} \mathscr{K}_{\alpha}^{2, \lambda+\sigma+2,0}(x, t ; \cdot, \cdot)=0$ on $H$. Hence, we have $u \in \boldsymbol{b}_{\alpha}^{2,1}(\lambda)$. Now, we show $l(u)=\Phi$. Let $v \in \tilde{\mathscr{B}}_{\alpha, 0}^{2}(\sigma)$. Since $\tilde{\mathscr{B}}_{\alpha, 0}^{2}(\sigma) \subset \tilde{\mathscr{B}}_{\alpha}^{2}(\sigma)$, Theorem 1 implies that $v=2^{\lambda+\sigma+2} P_{\alpha}^{\lambda+\sigma+1,1,(\sigma, 1)}\left(t^{\sigma+1} \mathscr{E}_{t} v\right)$ holds on $H$. Since $t^{\sigma+1} \mathscr{E}_{t} v \in C_{0}(H)$ by Lemma 11, we have

$$
\begin{aligned}
\Phi(v) & =2^{\lambda+\sigma+2} \Phi\left(P_{\alpha}^{\lambda+\sigma+1,1,(\sigma, 1)}\left(t^{\sigma+1} \mathscr{E}_{t} v\right)\right) \\
& =\Lambda\left(t^{\sigma+1} \mathscr{E}_{t} v\right)=\int_{H} \mathscr{E}_{t} v(x, t) t^{\sigma+1} d \mu(x, t) .
\end{aligned}
$$

On the other hand, the Fubini theorem, a differentiation under the integral sign, and Theorem 1 imply that

$$
\begin{aligned}
\langle u, v\rangle_{\lambda, \sigma} & \\
& =2^{\lambda+\sigma+2} \int_{H} u(y, s) \mathscr{E}_{t} v(y, s) s^{\lambda+\sigma+1} d V(y, s) \\
& =2^{\lambda+\sigma+2} \int_{H} \int_{H} \mathscr{K}_{\alpha}^{2, \lambda+\sigma+2,0}(x, t ; y, s) t^{\sigma+1} d \mu(x, t) \mathscr{E}_{t} v(y, s) s^{\lambda+\sigma+1} d V(y, s)
\end{aligned}
$$




$$
\begin{aligned}
& =\int_{H} 2^{\lambda+\sigma+2} \int_{H} \mathscr{K}_{\alpha}^{2, \lambda+\sigma+2,0}(x, t ; y, s) \mathscr{E}_{t} v(y, s) s^{\lambda+\sigma+1} d V(y, s) t^{\sigma+1} d \mu(x, t) \\
& =\int_{H} \mathscr{E}_{t}\left(2^{\lambda+\sigma+2} \int_{H} \mathscr{R}_{\alpha}^{\lambda+\sigma+1,1,1}(x, t ; y, s) \mathscr{E}_{t} v(y, s) s^{\lambda+\sigma+1} d V(y, s)\right) t^{\sigma+1} d \mu(x, t) \\
& =\int_{H} \mathscr{E}_{t} v(x, t) t^{\sigma+1} d \mu(x, t) .
\end{aligned}
$$

Consequently, we obtain $\imath(u)=\Phi$. This completes the proof.

\title{
References
}

[1] Y. Hishikawa, Fractional calculus on parabolic Bergman spaces, Hiroshima Math. J., 38 (2008), 471-488.

[2] Y. Hishikawa and M. Yamada, Function spaces of parabolic Bloch type, Hiroshima Math. J., 41 (2011), 55-87.

[3] Y. Hishikawa, M. Nishio and M. Yamada, $L^{(\alpha)}$-conjugates on parabolic Bergman spaces, Potential anal., 40 (2014), 525-537.

[4] Y. Hishikawa, M. Nishio and M. Yamada, A system of conjugate functions on parabolic Bloch spaces, J. Math. Soc. Japan, 70 (2018), 1085-1102.

[5] M. Nishio and K. Shimomura, Reproducing kernels for iterated parabolic operators on the upper half space with application to polyharmonic Bergman spaces, Complex Anal. Oper. Theory, 11 (2017), 1865-1878.

[6] M. Nishio and K. Shimomura, Reproducing property for iterated parabolic operators of fractional order, Math. Reports, 23(73), 1-2 (2021), 211-226.

[7] M. Nishio, N. Suzuki and M. Yamada, Toeplitz operators and Carleson measures on parabolic Bergman spaces, Hokkaido Math. J., 36 (2007), 563-583.

[ 8 ] M. Pavlović, Decompositions of $L^{p}$ and Hardy spaces of polyharmonic functions, J. Math. Anal. Appl., 216 (1997), 499-509.

[9] W. Ramey and H. Yi, Harmonic Bergman functions on half-spaces, Trans. Amer. Math. Soc., 348 (1996), 633-660.

[10] K. Tanaka, Biharmonic Bergman space and its reproducing kernel, Complex Var. Elliptic Equ., 63 (2018), 1642-1663.

\author{
Yôsuke Hishikawa \\ Depertment of Mathematics \\ Faculty of Education \\ Gifu University \\ 1-1 Yanagido 501-1193 Japan \\ E-mail:yhishik@gifu-u.ac.jp \\ Masaharu Nishio \\ College of Engineering \\ Chubu University
}


1200 Matsumoto, Kasugai 487-8501, Japan

E-mail: masaharunishio@isc.chubu.ac.jp

Katsunori Shimomura

Depertment of Mathematics

Faculty of Science

Ibaraki University

2-1-1 Bunkyo Mito 310-8512 Japan

E-mail: katsunori.shimomura.sci@vc.ibaraki.ac.jp

Masahiro Yamada

Depertment of Mathematics

Faculty of Education

Gifu University

1-1 Yanagido 501-1193 Japan

E-mail: yamada33@gifu-u.ac.jp 